\title{
On interpolative contractions that involve rational forms
}

Andreea Fulga ${ }^{1 *}$ (D)

Correspondence: afulga@unitbv.ro 1 Department of Mathematics and Computer Sciences, Transilvania University of Brasov, 500091, Brasov, Romania

\section{Springer}

\begin{abstract}
The aim of this paper is to investigate the interpolative contractions involving rational forms in the framework of $b$-metric spaces. We prove the existence of a fixed point of such a mapping with different combinations of the rational forms. A certain example is considered to indicate the validity of the observed result.
\end{abstract}

MSC: $47 \mathrm{H} 10 ; 54 \mathrm{H} 25 ; 46 \mathrm{~J} 10$

Keywords: Fixed point; Metric space; Interpolative contractions; b-metric

\section{Introduction and preliminaries}

It is worth noting that Caccioppoli [1] is the first author who extended the results of Banach [2] from normed space to metric space. After that, a number of authors have studied different abstract spaces to advance the Banach and Caccioppoli results. One of the successive generalizations was given Bakhtin [3] (and independently by Czerwik [4]) from metric space to b-metric space. Following this success, many authors have continued to work on this trend and reported several improvements, advances in the setting of $b$-metric spaces, see e.g. [5-12].

Let $x$ be a nonempty set and $6: x \times x \rightarrow[0,+\infty)$ be a metric on $x$. The notion of $b$-metric (reported in several papers, e.g., Bakhtin [3], Czerwik [4]) as an extension of a metric notion is obtained by replacing the triangle inequality of the metric with a general one

(B) $\quad b(u, \omega) \leq s[6(u, \mu)+b(\mu, \omega)]$ for every $u, \omega, \mu \in X$,

for fixed $s \geq 1$. The triplet $(X, b, s)$ is said to be a $b$-metric space. (It is worth pointing out that in case $s=1$ the space $(X, 6,1)$ coincides with a corresponding standard metric space.)

One of the basic examples for $b$-metric is the following.

Example ([5]) Let $(x, d)$ be a metric space. Then the function $6: x \times x \rightarrow[0,+\infty)$ defined as $b(u, \omega)=(d(u, \omega))^{p}$ with $p>1$ forms a $b$-metric (here $\left.s=2^{p-1}\right)$.

For more examples, see e.g. [5-12].

Like metric spaces, $b$-metric spaces admit a nice topology. On the other hand, alike metric, $b$-metric does not need to be continuous. For the sake of the integrity of the article, we recollect the basic topological notions here.

(c) The Author(s) 2021. This article is licensed under a Creative Commons Attribution 4.0 International License, which permits use, sharing, adaptation, distribution and reproduction in any medium or format, as long as you give appropriate credit to the original author(s) and the source, provide a link to the Creative Commons licence, and indicate if changes were made. The images or other third party material in this article are included in the article's Creative Commons licence, unless indicated otherwise in a credit line to the material. If material is not included in the article's Creative Commons licence and your intended use is not permitted by statutory regulation or exceeds the permitted use, you will need to obtain permission directly from the copyright holder. To view a copy of this licence, visit http://creativecommons.org/licenses/by/4.0/. 
We say that a sequence $\left\{u_{m}\right\}$ in a $b$-metric space $(X, b, s)$ is

(1) convergent to $u$ if $\lim _{n \rightarrow \infty} b\left(u_{m}, u\right)=0$. The limit of a convergent sequence is unique;

(2) Cauchy if $b\left(u_{m}, u_{n}\right) \rightarrow 0$ as $n, m \rightarrow \infty$.

Each convergent sequence in a $b$-metric space is Cauchy and, as usual, if each Cauchy sequence is convergent, then the $b$-metric space $(X, b, s)$ is said to be complete.

Definition 1.1 Let $(X, 6, s)$ be a $b$-metric space and $P: X \rightarrow X$ be a mapping. For $u_{0} \in X$, the orbit of $P$ at $u_{0}$ is the set

$$
\mathcal{O}\left(u_{0}, \mathcal{P}\right)=\left\{u_{0}, \mathcal{P} u_{0}, \mathcal{P}^{2} u_{0}, \ldots\right\} .
$$

The mapping $P$ is said to be orbitally continuous at a point $\varpi \in X$ if

$$
\lim _{j \rightarrow \infty} \mathcal{P}^{j} u_{0}=\varpi \text { implies } \quad \lim _{j \rightarrow \infty} \mathcal{P} \mathcal{P}^{j} u_{0}=\mathcal{P} \varpi .
$$

Additionally, if every Cauchy sequence $\left\{\mathcal{P}^{j} u_{0}\right\}$ is convergent in $X$, then the $b$-metric space $(X, 6, s)$ is said to be $P$-orbitally complete.

Definition $1.2([13])$ Let $(X, 6, s)$ be a $b$-metric space. We say that the mapping $P: X \rightarrow X$ is $m$-continuous, where $m=1,2, \ldots$, if $\lim _{m \rightarrow \infty} \mathcal{P}^{m} u_{n}=\mathcal{P} \varpi$, whenever the sequence $\left\{u_{n}\right\}$ in $X$ is such that $\lim _{m \rightarrow \infty} \mathcal{P}^{m-1} u_{n}=\varpi$.

Remark 1.3 We note that every continuous mapping is orbitally continuous in $X$ and also every complete $b$-metric space is $\mathcal{P}$-orbitally complete for any $\mathcal{P}: X \rightarrow X$, but the converse is not necessarily true.

On the other hand, it is clear that 1-continuity (which coincides with usual continuity) implies 2-continuity implies 3-continuity and so on, but the converse does not hold. Indeed, for example, considering the mapping $P: X \rightarrow X$, where $X=[0, \infty)$, defined by

$$
\mathcal{P}_{\mathcal{u}}= \begin{cases}5, & \text { if } u \in[0,5] \\ 1, & \text { if } u \in(5, \infty)\end{cases}
$$

we can easily see that $\mathcal{P}$ is not continuous (in $u=5$ ), but it is 2 -continuous because $\mathcal{P}^{2} u=5$.

Let us consider the following class of functions (named the set of b-comparison functions):

$$
\Theta=\left\{\phi:[0, \infty) \rightarrow[0, \infty) \mid \phi \text { is nondecreasing and } \sum_{n \geq 1} s^{n} \phi^{n}(\theta)<\infty \text { for each } \theta>0\right\},
$$

here $\phi^{n}$ represents the $n$th iterate of $\phi$. It can be shown that every function $\phi \in \Theta$ fulfills the following properties:

$(\phi 1) \phi(\theta)<\theta$ for any $\theta>0$;

$(\phi 2) \phi(0)=0$.

Let $X$ be a nonempty set and $\alpha: \mathcal{X} \times \mathcal{X} \rightarrow[0, \infty)$ be a function. We say that the mapping $\mathcal{P}: X \rightarrow X$ is $\alpha$-orbital admissible if

$$
\alpha\left(u, \mathcal{P}_{u}\right) \geq 1 \quad \text { implies } \quad \alpha\left(\mathcal{P}_{u}, \mathcal{P}^{2} u\right) \geq 1
$$

for all $u \in X$. 
Moreover, we say that the $b$-metric space $(X, 6, s)$ is $\alpha$-regular if for any sequence $\left\{\eta_{m}\right\}$ in $x$ such that $\lim _{m \rightarrow \infty} \eta_{m}=\eta$ and $\alpha\left(\eta_{m}, \eta_{m+1}\right) \geq 1$ we have $\alpha\left(\eta_{m}, \eta\right) \geq 1$.

(For more details and examples, see [14].)

Very recently, the notion of the interpolative contraction was introduced in [15]. The goal of this paper is to revisit the well-known Kannan type contraction in the setting of interpolation. After that, several famous contractions (Ćirić [16], Reich [17], Rus [18], Hardy- Rogers [19], Kannan [20], Bianchini [21]) are revisited in this new setting, see e.g. $[15,22-26]$

In this paper, we combine all these notions and trends to get more general results on the topic in the literature. We observe some interpolative contractions involving distinct rational forms that provide a fixed point in the framework of $b$-metric spaces.

\section{Main results}

Definition 2.1 Let $(X, 6, s)$ be a $b$-metric space. A self-mapping $P$ is called $\mathcal{A}_{P}^{l}$-admissible interpolative contraction $(l=1,2)$ if there exist $\phi \in \Theta$ and $\alpha: X \times X \rightarrow[0, \infty)$ such that

$$
\frac{1}{2} b(u, \mathcal{P} u) \leq 6(u, \omega) \quad \Rightarrow \quad \alpha(u, \omega) d(\mathcal{P} u, \mathcal{P} \omega) \leq \phi\left(\mathcal{A}_{\mathcal{P}}^{l}(u, \omega)\right),
$$

where $q_{i} \geq 0, i=1,2,3,4,5$, are such that $\sum_{i=1}^{5} q_{i}=1$ and

$$
\begin{aligned}
\mathcal{A}_{\mathcal{P}}^{1}(u, \omega)= & {[6(u, \omega)]^{q_{1}} \cdot[G(u, \mathcal{P} u)]^{q_{2}} } \\
& \cdot[\delta(\omega, \mathcal{P} \omega)]^{q_{3}} \cdot\left[\frac{6(\omega, \mathcal{P} \omega)(1+\sigma(u, \mathcal{P} u))}{1+6(u, \omega)}\right]^{q_{4}} \cdot\left[\frac{6(u, \mathcal{P} \omega)+b(\omega, \mathcal{P} u)}{2 s}\right]^{q_{5}},
\end{aligned}
$$

and

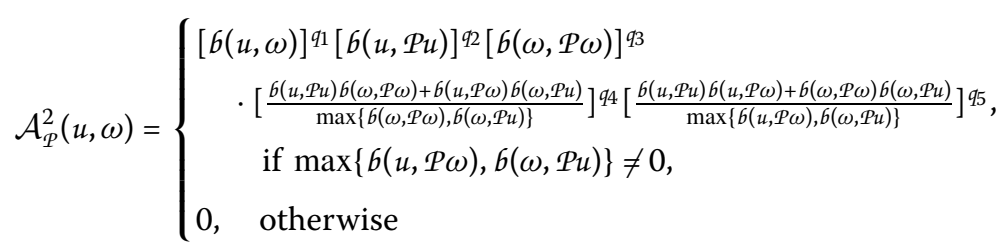

for any $u, \omega \in X \backslash \operatorname{Fix}_{\mathcal{P}}(X) .\left(\operatorname{Fix}_{\mathcal{P}}(X)=\{u \in \mathcal{X} \mid \mathcal{P} u=u\}.\right)$

The first main results of this paper is given in the following theorem.

Theorem 2.2 Let $(X, 6, s)$ be a complete b-metric space and $P$ be an $\mathcal{A}_{\mathcal{P}}^{1}$-admissible interpolative contraction such that

(i) $P$ is $\alpha$-orbital admissible;

(ii) there exists $u_{0} \in X$ such that $\alpha\left(u_{0}, \mathcal{P}_{u_{0}}\right) \geq 1$;

(iii $\left.i_{1}\right) \mathcal{P}$ is $m$-continuous for $m \geq 1$, or

(iii $\left.i_{2}\right) \mathcal{P}$ is orbitally continuous.

Then $\mathcal{P}$ possesses a fixed point $\varpi \in X$ and the sequence $\left\{\mathcal{P}^{m} u_{0}\right\}$ converges to this point $\varpi$.

Proof Let $u_{0}$ in $X$ be an arbitrary point and the sequence $\left\{\eta_{n}\right\}$ be defined as $\eta_{0}=u_{0}, \eta_{n}=$ $\mathcal{P}^{n} \eta_{0}$ for all $n \in \mathbb{N}$. If we can find some $q \in \mathbb{N}$ such that $\eta_{q}=\eta_{q+1}=\mathcal{P} \eta_{q}$, then it follows that 
$\eta_{q}$ is a fixed point of $\mathcal{P}$ and the proof is closed. For this reason, we can assume from now on that $\eta_{n} \neq \eta_{n-1}$ for any $n \in \mathbb{N}$. Using assumption $(i), P$ is $\alpha$-orbital admissible, we have

$$
\begin{aligned}
\alpha\left(\eta_{0}, \eta_{1}\right)=\alpha\left(\eta_{0}, P \eta_{0}\right) \geq 1 & \Rightarrow \alpha\left(\eta_{1}, \eta_{2}\right)=\alpha\left(\mathcal{P} \eta_{0}, P\left(P \eta_{0}\right)\right) \geq 1 \Rightarrow \cdots \\
& \Rightarrow \alpha\left(\eta_{n-1}, \eta_{n}\right) \geq 1
\end{aligned}
$$

On the other hand, we have that

$$
\frac{1}{2} b\left(\eta_{n-1}, P \eta_{n-1}\right)=\frac{1}{2} b\left(\eta_{n-1}, \eta_{n}\right) \leq 6\left(\eta_{n-1}, \eta_{n}\right) .
$$

Now, taking into account the main assumption that $P$ is an $\mathcal{A}_{P}^{1}$-admissible interpolative contraction, if we substitute $u$ with $\eta_{n-1}$ and $\omega$ with $\eta_{n}$ in (2.1), we get

$$
\begin{aligned}
& \zeta\left(\mathcal{P} u_{n-1}, \mathcal{P} u_{n}\right) \\
& \leq \alpha\left(\eta_{n-1}, \eta_{n}\right) 6\left(\mathcal{P} \eta_{n-1}, \mathcal{P} \eta_{n}\right) \leq \phi\left(\mathcal{A}_{\mathcal{P}}^{1}\left(\eta_{n-1}, \eta_{n}\right)\right) \\
& =\phi\left(\begin{array}{c}
{\left[6\left(\eta_{n-1}, \eta_{n}\right)\right]^{q_{1}} \cdot\left[6\left(\eta_{n-1}, \mathcal{P} \eta_{n-1}\right)\right]^{q_{2}} \cdot\left[6\left(\eta_{n}, \mathcal{P} \eta_{n}\right)\right]^{q_{3}}} \\
\cdot\left[\frac{6\left(\eta_{n}, P \eta_{n}\right)\left(1+6\left(\eta_{n-1}, P \eta_{n-1}\right)\right)}{1+6\left(\eta_{n-1}, \eta_{n}\right)}\right]^{q 4} \cdot\left[\frac{6\left(\eta_{n-1}, P \eta_{n}\right)+6\left(\eta_{n}, P_{n-1}\right)}{2 s}\right]^{q_{5}}
\end{array}\right) \\
& =\phi\left(\begin{array}{l}
{\left[6\left(\eta_{n-1}, \eta_{n}\right)\right]^{q_{1}} \cdot\left[6\left(\eta_{n-1}, \eta_{n}\right)\right]^{q_{2}} \cdot\left[6\left(\eta_{n}, \eta_{n+1}\right)\right]^{q_{3}}} \\
\cdot\left[\frac{6\left(\eta_{n}, \eta_{n+1}\right)\left(1+6\left(\eta_{n-1}, \eta_{n}\right)\right)}{1+6\left(\eta_{n-1}, \eta_{n}\right)}\right]^{q_{4}} \cdot\left[\frac{6\left(\eta_{n-1}, \eta_{n+1}\right)+6\left(\eta_{n}, \eta_{n}\right)}{2 s}\right]^{q_{5}}
\end{array}\right) \\
& =\phi\left(\left[6\left(\eta_{n-1}, \eta_{n}\right)\right]^{q_{1}+q_{2}} \cdot\left[6\left(\eta_{n}, \eta_{n+1}\right)\right]^{q_{3}+q_{4}} \cdot\left[\frac{6\left(\eta_{n-1}, \eta_{n+1}\right)}{2 s}\right]^{q_{5}}\right) \text {. }
\end{aligned}
$$

But by $(B)$, together with the monotony of the function $\phi$, it follows

$$
\begin{aligned}
& b\left(\eta_{n}, \eta_{n+1}\right) \\
& \quad=6\left(\mathcal{P} \eta_{n-1}, \mathcal{P} \eta_{n}\right) \\
& \quad \leq \phi\left(\left[6\left(\eta_{n-1}, \eta_{n}\right)\right]^{q_{1}+q_{2}} \cdot\left[6\left(\eta_{n}, \eta_{n+1}\right)\right]^{q_{3}+q_{4}} \cdot\left[\frac{6\left(\eta_{n-1}, \eta_{n}\right)+6\left(\eta_{n}, \eta_{n+1}\right)}{2}\right]^{q_{5}}\right)
\end{aligned}
$$

moreover, by $(\phi 1)$ we have

$$
6\left(\eta_{n}, \eta_{n+1}\right)<\left[6\left(\eta_{n-1}, \eta_{n}\right)\right]^{q_{1}+q_{2}} \cdot\left[6\left(\eta_{n}, \eta_{n+1}\right)\right]^{q_{3}+q_{4}} \cdot\left[\frac{6\left(\eta_{n-1}, \eta_{n}\right)+6\left(\eta_{n}, \eta_{n+1}\right)}{2}\right]^{q_{5}} .
$$

If there exists $m_{0} \in \mathbb{N}$ such that $6\left(\eta_{m_{0}-1}, \eta_{m_{0}}\right) \leq 6\left(\eta_{m_{0}}, \eta_{m_{0}+1}\right)$, then the above inequality becomes

$$
6\left(\eta_{m_{0}}, \eta_{m_{0}+1}\right)<\left[6\left(\eta_{m_{0}-1}, \eta_{m_{0}}\right)\right]^{q_{1}+q_{2}} \cdot\left[6\left(\eta_{m_{0}}, \eta_{m_{0}+1}\right)\right]^{q_{3}+q_{4}+q_{5}},
$$

which is a contradiction since (keeping in mind that $\left.1-\left(q_{3}+q_{4}+q_{5}\right)=q_{1}+q_{2}\right)$ it is equivalent with

$$
6\left(\eta_{m_{0}}, \eta_{m_{0}+1}\right)<6\left(\eta_{m_{0}-1}, \eta_{m_{0}}\right) .
$$


Therefore, for any $n \in \mathbb{N}$,

$$
6\left(\eta_{n}, \eta_{n+1}\right)<6\left(\eta_{n-1}, \eta_{n}\right)
$$

Furthermore, returning to inequality (2.5), we have

$$
6\left(\eta_{n}, \eta_{n+1}\right) \leq \phi\left(6\left(\eta_{n-1}, \eta_{n}\right)\right) \leq \cdots \leq \phi^{n}\left(6\left(\eta_{0}, \eta_{1}\right)\right) .
$$

Let $q \in \mathbb{N}$. Then, by $(B)$, together with (2.6), we obtain

$$
\begin{aligned}
6\left(\eta_{n}, \eta_{n+q}\right) & \leq s\left[6\left(\eta_{n}, \eta_{n+1}\right)+6\left(\eta_{n+1}, \eta_{n+q}\right)\right] \\
& \leq s 6\left(\eta_{n}, \eta_{n+1}\right)+s^{2} 6\left(\eta_{n+1}, \eta_{n+2}\right)+\cdots+s^{q} b\left(\eta_{n+q-1}, \eta_{n+q}\right) \\
& \leq s \phi^{n}\left(6\left(\eta_{0}, \eta_{1}\right)\right)+s^{2} \phi^{n+1}\left(6\left(\eta_{0}, \eta_{1}\right)\right)+\cdots+s^{q} \phi^{n+q-1}\left(6\left(\eta_{0}, \eta_{1}\right)\right) \\
& =\frac{1}{s^{n-1}} \sum_{j=n}^{n+q-1} s^{j} \phi^{j}\left(6\left(\eta_{0}, \eta_{1}\right)\right) \\
& \leq \frac{1}{s^{n-1}} \sum_{j=1}^{n+q-1} s^{j} \phi^{j}\left(6\left(\eta_{0}, \eta_{1}\right)\right) \rightarrow 0 \quad \text { as } q, n \rightarrow \infty .
\end{aligned}
$$

It follows that $\left\{\eta_{n}\right\}$ is a Cauchy sequence in a $\mathcal{P}$-orbitally complete $b$-metric space. Therefore, we can find $\varpi \in X$ such that $\lim _{n \rightarrow \infty} \mathcal{P}^{n} \eta_{0}=\varpi$.

We claim that $\varpi$ is a fixed point of the mapping $\mathcal{P}$ under of any hypothesis, $(i i i)_{1}$ or $(i i i)_{2}$. Indeed,

$$
\varpi=\lim _{n \rightarrow \infty} \eta_{n}=\lim _{n \rightarrow \infty} \mathcal{P}\left(\eta_{n-1}\right) .
$$

Moreover,

$$
\lim _{n \rightarrow \infty} \mathcal{P}^{m} \eta_{n}=\varpi \quad \text { for every } m \geq 1
$$

If $\mathcal{P}$ is $m$-continuous, then $\lim _{n \rightarrow \infty} \mathcal{P}^{m} \eta_{n}=\mathcal{P} \varpi$, and by (2.7) it follows that $\mathcal{P} \varpi=\varpi$.

If $\mathcal{P}$ is assumed to be orbitally continuous on $X$, then

$$
\varpi=\lim _{n \rightarrow \infty} \eta_{n-1}=\lim _{n \rightarrow \infty} \mathcal{P} \eta_{n-1}=\lim _{n \rightarrow \infty} \mathcal{P}\left(\mathcal{P}^{n-1} \eta_{0}\right)=\mathcal{P} \varpi
$$

Therefore, $\varpi \in \operatorname{Fix}_{\mathcal{P}}(X)$.

Example Let $x=[0,+\infty)$ and $b: x \times x \rightarrow[0,+\infty)$ be the $b$-metric defined as $6(u, \omega)=$ $(u-\omega)^{2}$ for all $u, \omega \in X$. Let the mapping $P: X \rightarrow X$ be defined by

$$
P(u)= \begin{cases}\frac{1}{2}, & \text { if } u \in[0,1), \\ \frac{u}{4}, & \text { if } u \in[1,2], \\ \frac{\sqrt{u^{2}+u+3}}{u^{2}+u+2}+\frac{\ln \left(u^{2}+u+2\right)}{u^{2}+u+4}, & \text { if } u \in(2,+\infty),\end{cases}
$$


and a function $\alpha: X \times X \rightarrow[0,+\infty)$, where

$$
\alpha(u, \omega)= \begin{cases}\sqrt{u^{2}+\omega^{2}+4}, & \text { if } u, \omega \in[0,1), \\ 3, & \text { if } u=0, \omega=2, \\ u^{2}+\omega / 3, & \text { if } u=\frac{1}{4}, \omega \in\{3,9\} \\ 0, & \text { otherwise. }\end{cases}
$$

Let also the comparison function $\phi:[0, \infty) \rightarrow[0, \infty), \phi(t)=t / 3$, and we choose $q_{1}=q_{5}=$ $1 / 10, q_{2}=q_{4}=1 / 5, q_{3}=2 / 5$. Thus, we can easily observe that assumptions (i) and (ii) are satisfied, and since $P^{2}(u)=1 / 2$ is continuous, assumption (iv) is also verified.

Case (i.) For $u, \omega \in[0,1]$, we have $\sigma(\mathcal{P} u, \mathcal{P} \omega)=0$, so inequality (2.1) holds.

Case (ii.) For $u=0$ and $\omega=2$, we have $\frac{1}{2} b\left(0, \frac{1}{2}\right)=\frac{1}{8}<4=b(0,2)$ and $b(\mathcal{P} u, \mathcal{P} \omega)=0$. Thus, (2.1) holds.

Case (iii.) For $u=1 / 4$ and $\omega=3$, we have $\frac{1}{2} b\left(0, \frac{1}{2}\right)=\frac{1}{8}<9=6(0,3) \Rightarrow$

$$
\alpha\left(\frac{1}{4}, 3\right) 6\left(\frac{1}{4}, 3\right)=0.003625861<0.534529784=\phi\left(\mathcal{A}_{P}^{1}\left(\frac{1}{4}, 3\right)\right) .
$$

Case (iv.) For $u=1 / 4$ and $\omega=9$, we have $\frac{1}{2} 6\left(0, \frac{1}{2}\right)=\frac{1}{8}<81=6(0,9) \Rightarrow$

$$
\alpha\left(\frac{1}{4}, 9\right) 6\left(\frac{1}{4}, 9\right)=0.368908954<2.453226625=\phi\left(\mathcal{A}_{P}^{1}\left(\frac{1}{4}, 9\right)\right) .
$$

All other cases are of no interest because $\alpha(u, \omega)=0$ and (2.1) is satisfied.

Therefore, the mapping $P$ is an $\mathcal{A}_{P}^{1}$-admissible interpolative contraction. On the other hand, since $P^{2}(u)=1 / 2$ is continuous and $P$ is $\alpha$-orbital continuous, by Theorem 2.2 we get that there exists a fixed point of the mapping $\mathcal{P}$; that is, $u=\frac{1}{2}$.

Theorem 2.3 Let $(X, 6, s)$ be a complete b-metric space and $\mathcal{P}$ be an $\mathcal{A}_{P}^{2}$-admissible interpolative contraction such that

(i) $\mathcal{P}$ is $\alpha$-orbital admissible;

(ii) there exists $u_{0} \in X$ such that $\alpha\left(u_{0}, \mathcal{P} u_{0}\right) \geq 1$;

(iii $\left.i_{1}\right) \mathcal{P}$ is $m$-continuous for $m \geq 1$, or

(iii $\left.i_{2}\right) \mathcal{P}$ is orbitally continuous.

Then $P$ possesses a fixed point $\varpi \in X$.

Proof As in the previous proof, for $u_{0} \in X$, we build the sequence $\left\{\eta_{n}\right\}$, where $\eta_{0}=u_{0}$ and $\eta_{n}=\mathcal{P} \eta_{n-1}=\mathcal{P}^{n} \eta_{0}$ for any $n \in \mathbb{N}$. Since $\eta_{n-1} \neq \eta_{n}$ for any $n \in \mathbb{N} \cup 0$, taking into account that the mapping $\mathcal{P}$ is supposed to be $\mathcal{A}_{\mathcal{P}}^{2}$-admissible interpolative contraction, we have

$$
\begin{aligned}
& \frac{1}{2} b\left(\eta_{n-1}, \mathcal{P} \eta_{n-1}\right)=\frac{1}{2} b\left(\eta_{n-1}, \eta_{n}\right) \leq 6\left(\eta_{n-1}, \eta_{n}\right) \quad \Rightarrow \\
& \alpha\left(\eta_{n-1}, \eta_{n}\right) \sigma\left(\mathcal{P} \eta_{n-1}, \mathcal{P} \eta_{n}\right) \leq \phi\left(\mathcal{A}_{\mathcal{P}}^{2}\left(\eta_{n-1}, \eta_{n}\right)\right),
\end{aligned}
$$

where

$$
\mathcal{A}_{\mathcal{P}}^{2}\left(\eta_{n-1}, \eta_{n}\right)=\left[6\left(\eta_{n-1}, \eta_{n}\right)\right]^{q_{1}} \cdot\left[6\left(\eta_{n-1}, \mathcal{P} \eta_{n-1}\right)\right]^{q_{2}} \cdot\left[6\left(\eta_{n}, \mathcal{P} \eta_{n}\right)\right]^{q_{3}}
$$




$$
\begin{aligned}
& \cdot\left[\frac{6\left(\eta_{n-1}, \mathcal{P} \eta_{n-1}\right) 6\left(\eta_{n}, \mathcal{P} \eta_{n}\right)+6\left(\eta_{n-1}, \mathcal{P} \eta_{n}\right) 6\left(\eta_{n}, \mathcal{P} \eta_{n-1}\right)}{\max \left\{6\left(\eta_{n}, \mathcal{P} \eta_{n}\right), 6\left(\eta_{n}, \mathcal{P} \eta_{n-1}\right)\right\}}\right]^{q_{4}} \\
& \cdot\left[\frac{6\left(\eta_{n-1}, \mathcal{P} \eta_{n-1}\right) 6\left(\eta_{n-1}, \mathcal{P} \eta_{n}\right)+6\left(\eta_{n}, \mathcal{P} \eta_{n}\right) 6\left(\eta_{n}, \mathcal{P} \eta_{n-1}\right)}{\max \left\{6\left(\eta_{n-1}, \mathcal{P} \eta_{n}\right), 6\left(\eta_{n}, \mathcal{P} \eta_{n-1}\right)\right\}}\right]^{q_{5}} \\
=[ & {\left[6\left(\eta_{n-1}, \eta_{n}\right)\right]^{q_{1}} \cdot\left[6\left(\eta_{n-1}, \eta_{n}\right)\right]^{q_{2}} \cdot\left[6\left(\eta_{n}, \eta_{n+1}\right)\right]^{q_{3}} } \\
& \cdot\left[\frac{6\left(\eta_{n-1}, \eta_{n}\right) 6\left(\eta_{n}, \eta_{n+1}\right)+6\left(\eta_{n-1}, \eta_{n+1}\right) 6\left(\eta_{n}, \eta_{n}\right)}{\max \left\{6\left(\eta_{n}, \eta_{n+1}\right), 6\left(\eta_{n}, \eta_{n}\right)\right\}}\right]^{q_{4}} \\
& \cdot\left[\frac{6\left(\eta_{n-1}, \eta_{n}\right) 6\left(\eta_{n-1}, \eta_{n+1}\right)+6\left(\eta_{n}, \eta_{n+1}\right) 6\left(\eta_{n}, \eta_{n}\right)}{\max \left\{6\left(\eta_{n-1}, \eta_{n+1}\right), 6\left(\eta_{n}, \eta_{n}\right)\right\}}\right]^{q_{5}} \\
= & {\left[6\left(\eta_{n-1}, \eta_{n}\right)\right]^{q_{1}+q_{2}} \cdot\left[6\left(\eta_{n}, \eta_{n+1}\right)\right]^{q_{3}} \cdot\left[\frac{6\left(\eta_{n-1}, \eta_{n}\right) 6\left(\eta_{n}, \eta_{n+1}\right)}{6\left(\eta_{n}, \eta_{n+1}\right)}\right]^{q_{4}} } \\
& \cdot\left[\frac{6\left(\eta_{n-1}, \eta_{n}\right) 6\left(\eta_{n-1}, \eta_{n+1}\right)}{6\left(\eta_{n-1}, \eta_{n+1}\right)}\right]^{q_{5}} \\
= & {\left[6\left(\eta_{n-1}, \eta_{n}\right)\right]^{q_{1}+q_{2}+q_{4}+q_{5}} \cdot\left[6\left(\eta_{n}, \eta_{n+1}\right)\right]^{q_{3}} . }
\end{aligned}
$$

Therefore, since by assumption $(i)$ it follows that $\alpha\left(\eta_{n-1}, \eta_{n}\right) \geq 1$ for all $n \in \mathbb{N}$, we have

$$
\begin{aligned}
6\left(\eta_{n}, \eta_{n+1}\right) & \leq \alpha\left(\eta_{n-1}, \eta_{n}\right) b\left(\mathcal{P} \eta_{n-1}, \mathcal{P} \eta_{n}\right) \leq \phi\left(\mathcal{A}_{\mathcal{P}}^{2}\left(\eta_{n-1}, \eta_{n}\right)\right) \\
& =\phi\left(\left[6\left(\eta_{n-1}, \eta_{n}\right)\right]^{q_{1}+q_{2}+q_{4}+q_{5}} \cdot\left[6\left(\eta_{n}, \eta_{n+1}\right)\right]^{q_{3}}\right) \\
& <\left[6\left(\eta_{n-1}, \eta_{n}\right)\right]^{q_{1}+q_{2}+q_{4}+q_{5}} \cdot\left[6\left(\eta_{n}, \eta_{n+1}\right)\right]^{q_{3}} .
\end{aligned}
$$

(Here, we used the property $(\phi 1)$ of the function $\phi$.

Thus,

$$
\left[6\left(\eta_{n}, \eta_{n+1}\right)\right]^{1-q_{3}}<\left[6\left(\eta_{n-1}, \eta_{n}\right)\right]^{q_{1}+q_{2}+q_{4}+q_{5}}=\left[6\left(\eta_{n-1}, \eta_{n}\right)\right]^{1-q_{3}},
$$

and then $6\left(\eta_{n}, \eta_{n+1}\right)<6\left(\eta_{n-1}, \eta_{n}\right)$ for any $n \in \mathbb{N}$. Furthermore, by $(2.8)$ and keeping in mind $(\phi 2)$, we obtain

$$
6\left(\eta_{n}, \eta_{n+1}\right)<\phi\left(6\left(\eta_{n-1}, \eta_{n}\right)\right)<\phi^{2}\left(6\left(\eta_{n-2}, \eta_{n-1}\right)\right)<\cdots<\phi^{n}\left(6\left(\eta_{0}, \eta_{1}\right)\right)
$$

and following the same steps as in the proof of Theorem 2.2, we can easily find that the sequence $\left\{\eta_{n}\right\}$ is Cauchy. Moreover, since $(X, 6, s)$ is supposed to be $\mathcal{P}$-orbitally complete, we can find a point $\varpi \in X$ such that $\lim _{n \rightarrow \infty} \mathcal{P}^{n} \eta_{0}=\varpi$. Assuming that $\mathscr{P}$ is $m$-continuous, we have

$$
\mathcal{P} \varpi=\lim _{n \rightarrow \infty} \mathcal{P}^{m} \eta_{n}=\lim _{n \rightarrow \infty} \eta_{n+m}=\varpi,
$$

and assuming that $\mathcal{P}$ is orbitally continuous, we get

$$
\mathcal{P} \varpi=\lim _{n \rightarrow \infty} \mathcal{P}\left(\mathcal{P}^{n} \eta_{0}\right)=\lim _{n \rightarrow \infty} \mathcal{P} \eta_{n}=\lim _{n \rightarrow \infty} \eta_{n+1}=\varpi,
$$

that is, $\varpi$ is a fixed point of $P$. 
In case we replace the continuity condition of the mapping with the continuity of the $b$-metric 6 , we get the following results.

Theorem 2.4 Let $(X, 6, s)$ be a complete, $\alpha$-regular $b$-metric space, where the $b$-metric 6 is continuous, and $P: X \rightarrow X$ is such that

$$
\frac{1}{2 s} 6(u, P u) \leq 6(u, \omega) \quad \Rightarrow \quad \alpha(u, \omega) d\left(\mathcal{P}_{u}, \mathcal{P} \omega\right) \leq \phi\left(\mathcal{A}_{\mathcal{P}}^{l}(u, \omega)\right),
$$

where $\phi \in \Theta$ and $\mathcal{A}_{p}^{l}$, for $l=1,2$ are given by (2.2) and (2.3). If

(i) $\mathcal{P}$ is $\alpha$-orbital admissible;

(ii) there exists $u_{0} \in X$ such that $\alpha\left(u_{0}, \mathcal{P} u_{0}\right) \geq 1$.

Then $P$ possesses a fixed point $\varpi \in X$, and the sequence $\left\{P^{m} u_{0}\right\}$ converges to this point $\varpi$.

Proof From the proof of Theorem 2.2 we know that the sequence $\left\{\eta_{n}\right\}$, where $\eta_{n}=P \eta_{n-1}=$ $\mathcal{P}^{n} \eta_{0}$ converges to a point $\varpi \in X$, and we claim that $\varpi$ is a fixed point of the mapping $\mathcal{P}$. For this purpose, we claim that

$$
\frac{1}{2 s} 6\left(\eta_{n}, \mathcal{P} \eta_{n}\right) \leq 6\left(\eta_{n}, \varpi\right)
$$

or

$$
\frac{1}{2 s} 6\left(\mathcal{P} \eta_{n}, \mathcal{P}\left(\mathcal{P} \eta_{n}\right)\right) \leq 6\left(\mathcal{P} \eta_{n}, \varpi\right) .
$$

Indeed, supposing the contrary

$$
\frac{1}{2 s} 6\left(\eta_{n}, \mathcal{P} \eta_{n}\right)>b\left(\eta_{n}, \varpi\right) \text { and } \frac{1}{2 s} \sigma\left(\mathcal{P} \eta_{n}, \mathcal{P}\left(\mathcal{P} \eta_{n}\right)\right)>\sigma\left(\mathcal{P} \eta_{n}, \varpi\right)
$$

we get that

$$
\begin{aligned}
b\left(\eta_{n}, \eta_{n+1}\right) & =b\left(\eta_{n}, \mathcal{P} \eta_{n}\right) \leq s\left[b\left(\eta_{n}, \varpi\right)+b\left(\varpi, \mathcal{P} \eta_{n}\right)\right] \\
& <s\left[\frac{1}{2 s} b\left(\eta_{n}, \mathcal{P} \eta_{n}\right)+\frac{1}{2 s} b\left(\mathcal{P} \eta_{n}, \mathcal{P}\left(\mathcal{P} \eta_{n}\right)\right)\right] \\
& =\frac{1}{2}\left[b\left(\eta_{n}, \eta_{n+1}\right)+b\left(\eta_{n+1}, \eta_{n+2}\right)\right] \leq 6\left(\eta_{n}, \eta_{n+1}\right) .
\end{aligned}
$$

This is a contradiction, and then (2.10) or (2.11) holds. Under the regularity assumption of the space $(X, 6, s)$, we have that $\alpha\left(\eta_{n}, \varpi\right) \geq 1$ for any $n \in \mathbb{N}$.

Case 1. $(l=1)$

(1.a) If (2.10) holds, we get

$$
\begin{aligned}
6\left(\eta_{n+1}, \mathcal{P} \varpi\right) \leq & \alpha\left(\eta_{n}, \varpi\right) b\left(\mathcal{P} \eta_{n}, \mathcal{P} \varpi\right) \leq \phi\left(\mathcal{A}_{\mathcal{P}}^{1}\left(\eta_{n}, \varpi\right)\right)<\mathcal{A}_{\mathcal{P}}^{1}\left(\eta_{n}, \varpi\right) \\
= & {\left[6\left(\eta_{n}, \varpi\right)\right]^{q_{1}} \cdot\left[6\left(\eta_{n}, \eta_{n+1}\right)\right]^{q_{2}} \cdot[6(\varpi, \mathcal{P} \varpi)]^{q_{3}} } \\
& \cdot\left[\frac{6(\varpi, \mathcal{P} \varpi)\left(1+6\left(\eta_{n}, \eta_{n+1}\right)\right)}{1+6\left(\eta_{n}, \varpi\right)}\right]^{q_{4}} \\
& \cdot\left[\frac{6\left(\eta_{n}, \mathcal{P} \varpi\right)+6\left(\varpi, \eta_{n+1}\right)}{2 s}\right]^{q_{5}} \cdot
\end{aligned}
$$


(1.b) If (2.11) holds,

$$
\begin{aligned}
6\left(\eta_{n+2}, \mathcal{P} \varpi\right) \leq & \alpha\left(\eta_{n+1}, \varpi\right) b\left(\mathcal{P}^{2} \eta_{n}, \mathcal{P} \varpi\right) \leq \phi\left(\mathcal{A}_{\mathcal{P}}^{1}\left(\mathcal{P} \eta_{n}, \varpi\right)\right)<\mathcal{A}_{\mathscr{P}}^{1}\left(\mathcal{P} \eta_{n}, \varpi\right) \\
= & {\left[6\left(\mathcal{P} \eta_{n}, \varpi\right)\right]^{q_{1}} \cdot\left[6\left(\eta_{n+1}, \eta_{n+2}\right)\right]^{q_{2}} \cdot[6(\varpi, \mathcal{P} \varpi)]^{q_{3}} } \\
& \cdot\left[\frac{6(\varpi, \mathcal{P} \varpi)\left(1+6\left(\eta_{n+1}, \eta_{n+2}\right)\right)}{1+6\left(\eta_{n+1}, \varpi\right)}\right]^{q_{4}} \\
& \cdot\left[\frac{6\left(\eta_{n+1}, \mathcal{P} \varpi\right)+6\left(\varpi, \eta_{n+2}\right)}{2 s}\right]^{q_{5}} \cdot
\end{aligned}
$$

We can distinguish the following two situations:

(i) $q_{1}+q_{2}>0$.

Letting $n \rightarrow \infty$ in (2.12) respectively (2.13), we obtain $6(\varpi, P \varpi)=0$. Thus, $\mathcal{P} \varpi=\varpi$.

(ii) $q_{1}=q_{2}=0$.

In this case, when $n \rightarrow \infty$, from (2.12), (2.13) and keeping in mind the continuity of $b$-metric 6 , we get

$$
6(\varpi, \mathcal{P} \varpi)<[6(\varpi, \mathcal{P} \varpi)]^{q_{3}+q_{4}+q_{5}}=6(\varpi, \mathcal{P} \varpi),
$$

which is a contradiction.

Consequently, $\mathscr{P} \varpi=\varpi$, that is, $\varpi$ is a fixed point of the mapping $\mathcal{P}$.

Case 2. $(l=2)$

(2.a) If (2.10) holds, we get

$$
\begin{aligned}
& 6\left(\eta_{n+1}, \mathcal{P} \varpi\right) \leq \alpha\left(\eta_{n}, \varpi\right) 6\left(\mathcal{P} \eta_{n}, \mathcal{P} \varpi\right) \leq \phi\left(\mathcal{A}_{P}^{2}\left(\eta_{n}, \varpi\right)\right)<\mathcal{A}_{P}^{2}\left(\eta_{n}, \varpi\right) \\
& =\left[6\left(\eta_{n}, \varpi\right)\right]^{q_{1}} \cdot\left[6\left(\eta_{n}, \eta_{n+1}\right)\right]^{q_{2}} \cdot[6(\varpi, \mathcal{P} \varpi)]^{q_{3}} \\
& \cdot\left[\frac{6(\varpi, \mathcal{P} \varpi) 6\left(\eta_{n}, \eta_{n+1}\right)+6\left(\varpi, \eta_{n+1}\right) 6\left(\eta_{n}, \mathcal{P} \varpi\right)}{\max \left\{6\left(\eta_{n}, \eta_{n+1}\right), 6\left(\eta_{n+1}, \mathcal{P} \varpi\right)\right\}}\right]^{q_{4}} \\
& \cdot\left[\frac{6(\varpi, \mathcal{P} \varpi) 6\left(\varpi, \eta_{n+1}\right)+b\left(\eta_{n}, \eta_{n+1}\right) b\left(\eta_{n}, \mathcal{P} \varpi\right)}{\max \left\{6\left(\varpi, \eta_{n+1}\right), 6\left(\eta_{n+1}, \mathcal{P} \varpi\right)\right\}}\right]^{q_{5}} \text {. }
\end{aligned}
$$

(2.b) If (2.11) holds,

$$
\begin{aligned}
6\left(\eta_{n+2}, \mathcal{P} \varpi\right) \leq & \alpha\left(\eta_{n+1}, \varpi\right) 6\left(\mathcal{P}^{2} \eta_{n}, \mathcal{P} \varpi\right) \leq \phi\left(\mathcal{A}_{\mathcal{P}}^{2}\left(\mathcal{P} \eta_{n}, \varpi\right)\right)<\mathcal{A}_{\mathcal{P}}^{2}\left(\mathcal{P} \eta_{n}, \varpi\right) \\
= & {\left[6\left(\eta_{n+1}, \varpi\right)\right]^{q_{1}} \cdot\left[6\left(\eta_{n+1}, \eta_{n+2}\right)\right]^{q_{2}} \cdot[6(\varpi, \mathcal{P} \varpi)]^{q_{3}} } \\
& \cdot\left[\frac{6(\varpi, \mathcal{P} \varpi) 6\left(\eta_{n+1}, \eta_{n+2}\right)+6\left(\varpi, \eta_{n+2}\right) 6\left(\eta_{n+1}, \mathcal{P} \varpi\right)}{\max \left\{b\left(\eta_{n+1}, \eta_{n+2}\right), 6\left(\eta_{n+2}, \mathcal{P} \varpi\right)\right\}}\right]^{q_{4}} \\
& \cdot\left[\frac{6(\varpi, \mathcal{P} \varpi) 6\left(\varpi, \eta_{n+2}\right)+6\left(\eta_{n+1}, \eta_{n+2}\right) 6\left(\eta_{n+1}, \mathcal{P} \varpi\right)}{\max \left\{6\left(\varpi, \eta_{n+2}\right), 6\left(\eta_{n+2}, \mathcal{P} \varpi\right)\right\}}\right]^{q_{5}} .
\end{aligned}
$$

We can distinguish the following two situations:

(i) $q_{1}+q_{2}+q_{4}+q_{5}>0$.

Letting $n \rightarrow \infty$ in (2.14), respectively (2.15), we obtain $b(\varpi, P \varpi)=0$. Thus, $\mathscr{P} \varpi=\varpi$. 
(ii) $q_{1}=q_{2}=q_{4}=q_{5}=0$.

In this case, when $n \rightarrow \infty$, from (2.14) and (2.15), we get

$$
6(\varpi, \mathcal{P} \varpi)<[6(\varpi, \mathcal{P} \varpi)]^{q_{3}}=6(\varpi, \mathcal{P} \varpi),
$$

which is a contradiction.

Consequently, $\mathcal{P} \varpi=\varpi$, that is, $\varpi$ is a fixed point of the mapping $\mathcal{P}$.

Example Let $x=\{1,2,3,5\}$ and $6: x \times X \rightarrow[0,+\infty)$ be a $b$-metric space $(s=2)$, defined by

$$
\sigma(u, \omega)= \begin{cases}(u+\omega)^{2}, & \text { if } u \neq \omega \\ 0, & \text { if } u=\omega\end{cases}
$$

Let $P$ be a self-mapping on $X$, with $P 1=P 5=1$ and $P 2=P 3=2$. Taking $\alpha: X \times X \rightarrow$ $[0,+\infty), \alpha(u, \omega)=2$ for all $u, \omega \in X, \phi(t)=t / 2$ and the constants $q_{i}=\frac{1}{5}$ for $i \in\{1,2,3,4,5\}$, we have

$$
\begin{aligned}
& \frac{1}{2 s} 6(3, P 3)=\frac{25}{4}<64=6(3,5) \Rightarrow \\
& \alpha(3,5) 6(P 3, P 5)=18<19.37742=\phi\left(\mathcal{A}_{P}^{2}(3,5)\right) .
\end{aligned}
$$

Thus, by Theorem 2.4, the mapping $\mathcal{P}$ has (at least) a fixed point.

\section{Consequences}

Corollary 3.1 Let $(X, 6, s)$ be a complete $b$-metric space and $P: X \rightarrow X$ be a mapping such that

$$
\alpha(u, \omega) \mathscr{b}(\mathcal{P} u, \mathcal{P} \omega) \leq \phi\left(\mathcal{A}_{\mathcal{P}}^{l}(u, \omega)\right)
$$

for any $u, \omega \in X \backslash \operatorname{Fix}_{\mathcal{P}}(X)$, where $\mathcal{A}_{p}^{l}, l=1,2$, are defined by (2.2) and (2.3) and $\phi \in \Theta$. Then $P$ possesses a fixed point $\varpi \in X$ provided that

(i) $\mathcal{P}$ is $\alpha$-orbital admissible;

(ii) there exists $u_{0} \in X$ such that $\alpha\left(u_{0}, \mathcal{P} u_{0}\right) \geq 1$;

(iii $\left.i_{1}\right) \mathcal{P}$ is $m$-continuous for $m \geq 1$, or

(iii $\left.i_{2}\right) \mathcal{P}$ is orbitally continuous.

Corollary 3.2 Let $(X, 6, s)$ be a complete $b$-metric space and $P: X \rightarrow X$ be a mapping such that

$$
\frac{1}{2} 6(u, \mathcal{P} u) \leq 6(u, \omega) \quad \Rightarrow \quad 6(\mathcal{P} u, \mathcal{P} \omega) \leq \phi\left(\mathcal{A}_{\mathcal{P}}^{l}(u, \omega)\right)
$$

for any $u, \omega \in X \backslash \operatorname{Fix}_{\mathcal{P}}(X)$, where $\mathcal{A}_{\mathcal{P}}^{l}, l=1,2$, are defined by (2.2) and (2.3). Then $\mathcal{P}$ possesses a fixed point $\varpi \in X$, provided that either $P$ is m-continuous for $m \geq 1$ or $\mathcal{P}$ is orbitally continuous.

Proof Put $\alpha(u, \omega)=1$ in Theorem 2.2, respectively 2.3. 
Corollary 3.3 Let $(X, 6, s)$ be a complete $b$-metric space and $\mathcal{P}: X \rightarrow X$ be a mapping such that there exists $\kappa \in[0,1)$ such that

$$
\frac{1}{2} \sigma(u, \mathcal{P} u) \leq \sigma(u, \omega) \quad \Rightarrow \quad \sigma\left(\mathcal{P}_{u}, \mathcal{P} \omega\right) \leq \kappa \cdot \mathcal{A}_{\mathcal{P}}^{l}(u, \omega)
$$

for any $u, \omega \in X \backslash \operatorname{Fix}_{P}(X)$, where $\mathcal{A}_{P}^{l}, l=1,2$, are defined by (2.2) and (2.3). Then $P$ possesses a fixed point $\varpi \in X$, provided that either $\mathcal{P}$ is m-continuous for $m \geq 1$, or $\mathcal{P}$ is orbitally continuous.

Proof Put $\phi(t)=\kappa \cdot t$ in Corollary 3.2.

Corollary 3.4 Let $(X, 6, s)$ be a complete $b$-metric space such that 6 is continuous. A mapping $\mathcal{P}: X \rightarrow X$ has a fixed point in $X$ provided that

$$
\frac{1}{2 s} 6(u, \mathcal{P} u) \leq b(u, \omega) \quad \Rightarrow \quad b\left(\mathcal{P}_{u}, \mathcal{P} \omega\right) \leq \phi\left(\mathcal{A}_{\mathcal{P}}^{l}(u, \omega)\right),
$$

where $\phi \in \Theta$ and $\mathcal{A}_{p}^{l}$, for $l=1,2$ are given by (2.2) and (2.3).

Proof Put $\alpha(u, \omega)=1$ in Theorem 2.4.

Corollary 3.5 Let $(X, 6, s)$ be a complete b-metric space such that 6 is continuous. A mapping $P: X \rightarrow X$ has a fixed point in $X$ provided that there exists $\kappa \in[0,1)$ such that

$$
\frac{1}{2 s} 6\left(u, \mathcal{P}_{u}\right) \leq 6(u, \omega) \quad \Rightarrow \quad \sigma\left(\mathcal{P}_{u}, \mathcal{P} \omega\right) \leq \kappa \mathcal{A}_{P}^{l}(u, \omega),
$$

where $\mathcal{A}_{p}^{l}$ for $l=1,2$ are given by (2.2) and (2.3).

Proof Put $\phi(t)=\kappa \cdot t$ in Corollary 3.4.

\section{Acknowledgements}

The author thanks the anonymous referees for their outstanding comments, suggestions, and ideas that helped improve this work.

Funding

This study did not receive funding.

Availability of data and materials

No data was generated or used during the study; this paper was not about data.

Competing interests

The author declares that they have no competing interests.

Authors' contributions

All authors read and approved the final manuscript.

\section{Publisher's Note}

Springer Nature remains neutral with regard to jurisdictional claims in published maps and institutional affiliations.

Received: 9 August 2021 Accepted: 27 September 2021 Published online: 12 October 2021 


\section{References}

1. Caccioppoli, R.: Una teorema generale sull'esistenza di elementi uniti in una transformazione funzionale. Rend. Accad. Naz. Lincei 11, 794-799 (1930)

2. Banach, S.: Sur les opérations dans les ensembles abstraits et leur application aux équations intégrales. Fundam. Math. 3, 133-181 (1922)

3. Bakhtin, I.A.: The contraction mapping principle in quasimetric spaces. Funct. Anal. Unianowsk Gos. Ped. Inst. 30 26-37 (1989)

4. Czerwik, S.: Contraction mappings in b-metric spaces. Acta Math. Inform. Univ. Ostrav. 1, 5-11 (1993)

5. Karapınar, E.: A short survey on the recent fixed point results on b-metric spaces. Constr. Math. Anal. 1(1), 15-44 (2018)

6. Alghamdi, M.A., Gulyaz-Ozyurt, S., Karapınar, E.: A note on extended Z-contraction. Mathematics 8, 195 (2020)

7. Afshari, H., Rezapour, Sh., Shahzad, N.: Absolute retract of the common fixed points set of two multifunctions. Topol. Methods Nonlinear Anal. 40, 429-436 (2012)

8. Aydi, H., Karapınar, E., Bota, M.F., Mitrovic, S.: A fixed point theorem for set-valued quasi-contractions in $b$-metric spaces. Fixed Point Theory Appl. 2012, 88 (2012)

9. Afshari, H., Aydi, H.: Existence and approximative fixed points for multifunctions. Asian-Eur. J. Math. 12, 1950022 (2019)

10. Aydi, H., Bota, M.F., Karapınar, E., Moradi, S.: A common fixed point for weak phi-contractions on b-metric spaces. Fixed Point Theory 13(2), 337-346 (2012)

11. Afshari, H., Alsulami, H.H., Karapınar, E.: On the extended multivalued Geraghty type contractions. J. Nonlinear Sci. Appl. 9, $46954706(2016)$

12. Afshari, H., Aydi, H., Karapınar, E.: On generalized $\alpha-\psi$-Geraghty contractions on $b$-metric spaces. Georgian Math. J. 27, 9-21 (2020)

13. Pant, A., Pant, R.P.: Fixed points and continuity of contractive maps. Filomat 31(11), 3501-3506 (2017)

14. Popescu, O.: Some new fixed point theorems for $\alpha$-Geraghty contractive type maps in metric spaces. Fixed Point Theory Appl. 2014, 190 (2014)

15. Karapınar, E.: Revisiting the Kannan type contractions via interpolation. Adv. Theory Nonlinear Anal. Appl. 2, 85-87 (2018)

16. Ćirić, Lj:: A generalization of Banach's contraction principle. Proc. Am. Math. Soc. 45, 267-273 (1974)

17. Reich, S.: Some remarks concerning contraction mappings. Can. Math. Bull. 14, 121-124 (1971)

18. Rus, I.A.: Generalized Contractions and Applications. Cluj University Press, Clui-Napoca (2001)

19. Hardy, G.E., Rogers, T.D.: A generalization of a fixed point theorem of Reich. Can. Math. Bull. 16, $201-206$ (1973)

20. Kannan, R.: Some results on fixed points. Bull. Calcutta Math. Soc. 60, 71-76 (1968)

21. Bianchini, R.M., Grandolfi, M.: Transformazioni di tipo contracttivo generalizzato in uno spazio metrico. Atti Accad. Naz. Lincei, VII. Ser. Rend. Cl. Sci. Fis. Mat. Natur. 45, 212-216 (1968)

22. Agarwal, R.P., Karapınar, E.: Interpolative Rus-Reich-Ciric type contractions via simulation functions. An. Ştiinţ. Univ. 'Ovidius' Constanţa, Ser. Mat. 27(3), 137-152 (2019)

23. Aydi, H., Chen, C.M., Karapınar, E.: Interpolative Ciric-Reich-Rus type contractions via the Branciari distance. Mathematics 7(1), 84 (2019)

24. Aydi, H., Karapınar, E., de Hierro, A.F.R.L.: $\omega$-Interpolative Ciric-Reich-Rus-type contractions. Mathematics 7, 57 (2019)

25. Karapınar, E., Alqahtani, O., Aydi, H.: On interpolative Hardy-Rogers type contractions. Symmetry 11(1), 8 (2019)

26. Karapınar, E., Agarwal, R., Aydi, H.: Interpolative Reich-Rus-Ćirić type contractions on partial metric spaces. Mathematics 6, 256 (2018) https://doi.org/10.3390/math6110256

\section{Submit your manuscript to a SpringerOpen ${ }^{\circ}$ journal and benefit from:}

- Convenient online submission

- Rigorous peer review

- Open access: articles freely available online

- High visibility within the field

- Retaining the copyright to your article

Submit your next manuscript at $\boldsymbol{s p r i n g e r o p e n . c o m ~}$ 